\title{
ABOUT BERGSTRÖM'S INEQUALITY
}

\section{OVIDIU T. POP}

Abstract. In this paper, we generalize identity (3), from where we obtain a rafinement of inequalities (1) and (2).

Mathematics subject classification (2000): 26D15.

Keywords and phrases: Bergström's inequality, Lagrange's identity, multivariate inequalities refinements.

\section{REFERENCES}

[1] G. Hardy, J. E. Littlewood and G. Pólya, Inequalities, Cambridge, 1934.

[2] E. F. Bechencbach AND R. Bellman, Inequalities, Springer, Berlin, Göttingen and Heidelberg, 1961.

[3] R. Bellman, Notes on Matrix Theory - IV (An Inequality Due to Bergström), Amer. Math. Montly, Vol. 62 (1955), 172-173

[4] H. BergStröm, A triangle inequality for matrices, Den Elfte Skandinaviske Matematikerkongress, 1949, Trodheim, Johan Grundt Tanums Forlag, Oslo, 1952, 264-267.

[5] D. Mărghidanu, J. L. Díaz-Barrero And S. RĂdulescu, New refinements of some classical inequalities, to appear in Math. Ineq.\& Appl.

[6] D. S. Mitrinović, Analytic Inequalities, Springer-Verlag, Berlin, 1970. 